Article

\title{
Simulation of Image Performance Characteristics of the Landsat Data Continuity Mission (LDCM) Thermal Infrared Sensor (TIRS)
}

\section{John Schott ${ }^{1}{ }^{*}$, Aaron Gerace ${ }^{1}$, Scott Brown ${ }^{1}$, Michael Gartley ${ }^{1}$, Matthew Montanaro ${ }^{2}$ and Dennis C. Reuter $^{3}$}

1 Center for Imaging Science, Rochester Institute of Technology, 54 Lomb Memorial Drive, Rochester, NY 14623, USA; E-Mails: gerace@ cis.rit.edu (A.G); brown@ cis.rit.edu (S.B.); gartley@ cis.rit.edu (M.G.)

2 Sigma Space Corporation, NASA Goddard Space Flight Center, 8800 Greenbelt Road, Greenbelt, MD 20771, USA; E-Mail: matthew.montanaro@nasa.gov

3 NASA Goddard Space Flight Center, 8800 Greenbelt Road, Greenbelt, MD 20771, USA;

E-Mail: Dennis.C.Reuter@mail.nasa.gov

* Author to whom correspondence should be addressed; E-Mail: schott@cis.rit.edu;

Tel.: +1-585-475-5170; Fax: +1-585-475-5988.

Received: 6 July 2012; in revised form: 15 August 2012 / Accepted: 16 August 2012 /

Published: 22 August 2012

\begin{abstract}
The next Landsat satellite, which is scheduled for launch in early 2013, will carry two instruments: the Operational Land Imager (OLI) and the Thermal Infrared Sensor (TIRS). Significant design changes over previous Landsat instruments have been made to these sensors to potentially enhance the quality of Landsat image data. TIRS, which is the focus of this study, is a dual-band instrument that uses a push-broom style architecture to collect data. To help understand the impact of design trades during instrument build, an effort was initiated to model TIRS imagery. The Digital Imaging and Remote Sensing Image Generation (DIRSIG) tool was used to produce synthetic "on-orbit" TIRS data with detailed radiometric, geometric, and digital image characteristics. This work presents several studies that used DIRSIG simulated TIRS data to test the impact of engineering performance data on image quality in an effort to determine if the image data meet specifications or, in the event that they do not, to determine if the resulting image data are still acceptable.
\end{abstract}


Keywords: Landsat; TIRS; thermal; DIRSIG; simulated imagery; image quality

\section{Introduction}

The next Landsat satellite, referred to as LDCM or Landsat 8, is scheduled for launch in early 2013 and will carry two instruments. The Operational Land Imager (OLI) is a push-broom multispectral sensor designed to continue or expand on the Landsat Enhanced Thematic Mapper Plus (ETM+) reflective imaging capabilities [1]. The Thermal Infrared Sensor (TIRS) is a two band push-broom thermal infrared sensor designed to continue or enhance the Landsat ETM+ thermal imaging capabilities [2]. The TIRS instrument, which is the focus of this study, represents a significant departure from previous Landsat thermal sensors. First it is a separate instrument whose data are to be combined with the OLI data to form a registered image data set. In addition, it is a push-broom design using three two-dimensional array Quantum Well Infrared Photodetector (QWIP) detectors to span the cross-track direction [3]. Filters are used to allow acquisition of two spectral bands (see Figure 1). In addition to these fundamental changes, TIRS uses refractive optics instead of ETM+'s reflective optics and a single full aperture radiometric calibrator and a deep space view which can be periodically introduced instead of ETM+'s dual source internal calibrator which is monitored at the beginning and end of every line of data.

These differences from the ETM+ design will potentially result in a number of changes in the character of the Landsat 8 image data. To better understand the impact of design trades during instrument build and test and to evaluate image processing algorithms and image quality issues in flight, an effort was initiated to model TIRS images. The goal of this modeling effort is to produce synthetic data streams that have the detailed radiometric, geometric, and digital image characteristics that TIRS data will have. As described in Section 2, these simulated data can then be used to test the impact of engineering performance data on image quality, to determine if the image data meet specifications or, if they do not, to determine if the resulting image data are still acceptable. Similar studies will be conducted to support the NASA/USGS image assessment teams as they identify and attempt to compensate for anomalies and changes in instrument performance during operation on orbit. The Digital Imaging and Remote Sensing Image Generation (DIRSIG) modeling tool is being used for these simulations. The DIRSIG tool has been extensively developed to simulate the spectral radiance images produced by sensors that observe the reflective and emitted energy from the Earth's surface [4,5]. DIRSIG incorporates solar irradiance, sky light illumination, atmospheric propagation, thermal models, self emission, reflection of thermal downwelled radiance and scattered and emitted path radiance. To support the Landsat sensor modeling effort described here, a number of improvements to the DIRSIG sensor modeling tools were developed to produce high fidelity simulation of the Landsat 8 instruments. 
Figure 1. Illustration of the Thermal Infrared Sensor (TIRS) focal plane layout. Three Quantum Well Infrared Photodetector (QWIP) arrays containing 512 by 640 detectors make up the focal plane. Spectral filters and masks are placed over specific areas of each QWIP to produce the two TIRS spectral channels and the blind band. Data from the filtered regions on the three QWIP arrays are combined to produce an effective across-track linear imaging array of 1,850 detectors per band by discarding approximately 7 columns on either end of the line and allowing for approximately 28 columns of overlap between adjacent arrays.

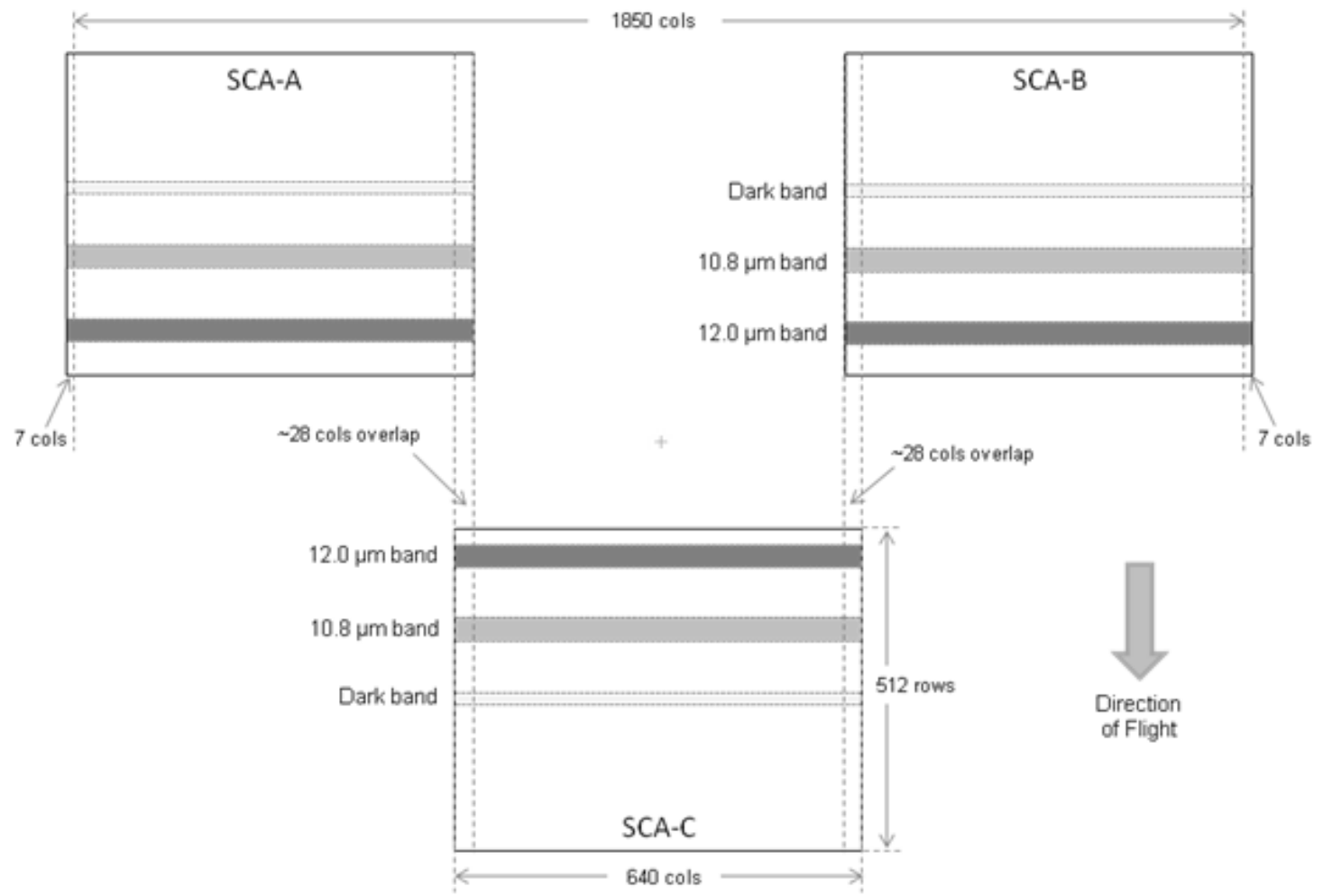

\section{Results and Discussion}

\subsection{Orbit, Earth Geometry and External Sensor Models}

In order to effectively model the Landsat image acquisition geometry, the DIRSIG model was modified to incorporate dynamic motion of the spacecraft as described by NORAD Two Line Element (TLE) orbital descriptors. Using the simplified general perturbation version 4 (SGP4) code [6] the satellite location and velocity vectors at each sample time desired (e.g., for each clock cycle of the detector read electronics) are produced from the TLE data. In order to take advantage of this orbital data, the DIRSIG scene geometry was upgraded to incorporate descriptions of the Earth that include the WGS 84 Geoid geometry with digital elevation maps (DEMs) superimposed, see Figure 2.

Note that because the various bands and arrays "look" forward or aft at different view angles, the parallax shifts are different for targets at different target-sensor slant paths (elevations). This means that all the appropriate acquisition geometry has to be properly modeled in DIRSIG to introduce the actual amount of band-to-band misregistration in the "raw" data stream sensed by the simulated system. These band-to-band registration issues are further complicated for Landsat 8 because the OLI and TIRS sensors, while on the same platform, have separate lines of sight and the line of sight 
variation induced by deck warping and jitter will differ for the two instruments. In order to account for these, the DIRSIG models account for offsets in the mounting locations and independent external geometry descriptors and jitter models for the two instruments [7].

Figure 2. Illustration of how a digital elevation map (DEM) is superimposed on the Geoid to form a 3D surface that can be facetized to form a "scene" in Digital Imaging and Remote Sensing Image Generation (DIRSIG).

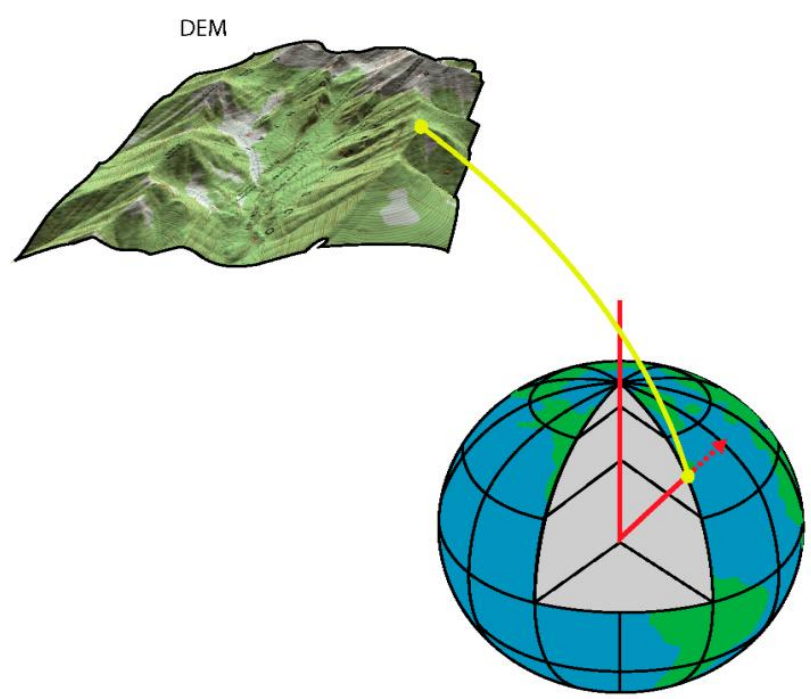

DEM is height above or below WGS 84 .

\subsection{Internal Geometry Models}

The internal geometry models for a TIRS type sensor are complicated from "text book" push-broom geometry by a number of factors. First in order to align the 3 QWIP arrays and account for read out electronics, the arrays must be offset from each other in the "in-track" direction (see Figure 1). In addition, the arrays are not perfectly aligned with each other or with the "cross-track" direction. Finally optical distortion causes the projection of an image to shift differently at different locations on the focal plane (e.g., barrel or pin cushion distortion). To account for these distortions, DIRSIG was modified to enable the angular location from the optical axis of the center of each detector element to be input in table form for each spectral band (see Figure 3).

For devices such as TIRS, where different rows or even detectors can be selected for imaging and downlink, a selectable look-up table of detector locations can be implemented [7]. For the TIRS study, the detector location information was acquired using geometric camera calibration data acquired during the instrument environmental testing. This location information can also be used to control detector sampling times and read out to generate data acquisition and readout data that mimic actual data streams. 
Figure 3. Illustration of angle-angle lines of sight relative to the optical axis for each detector element (left). The complexity of the focal plane and how this is converted to an angle-angle look-up table (in this case for OLI) are shown at the right.
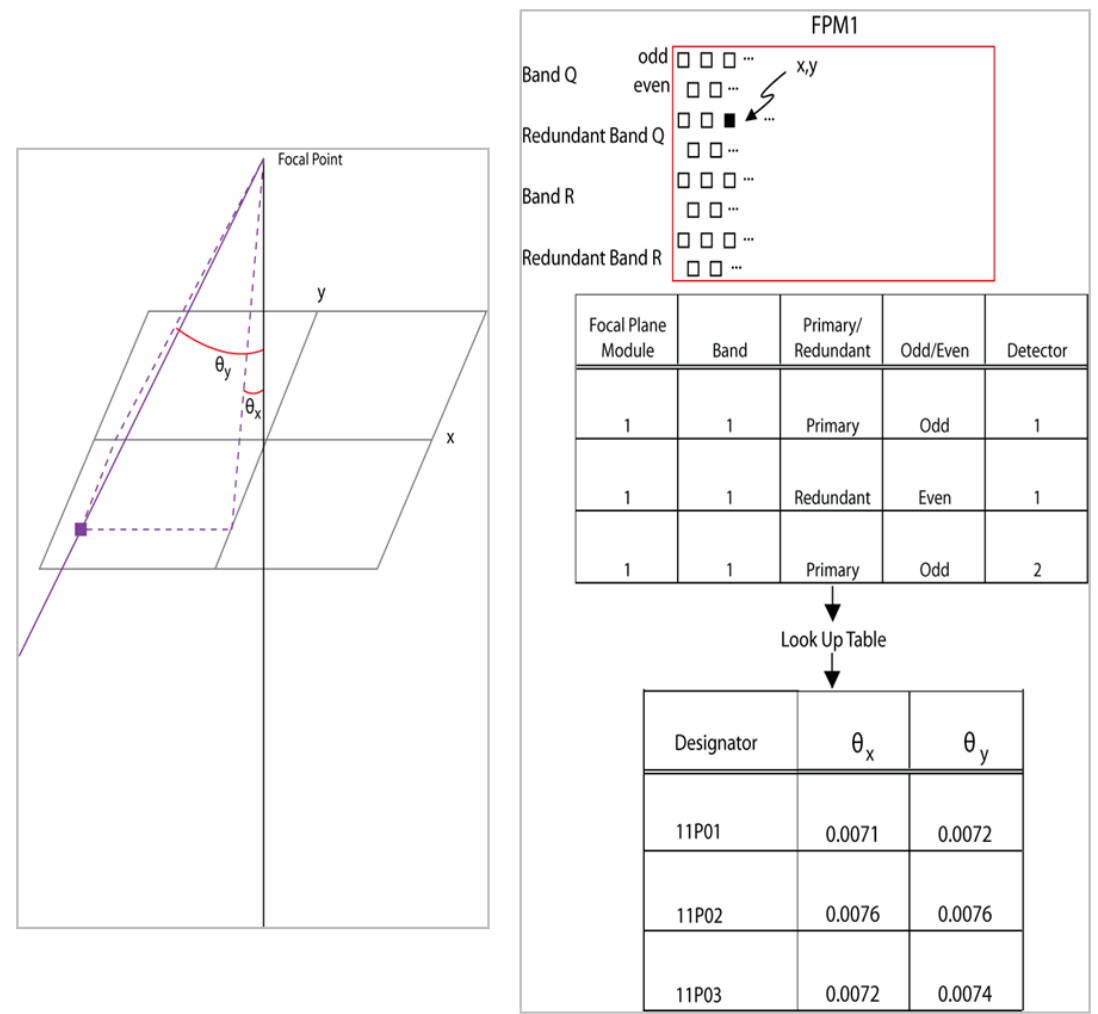

\subsection{Simulated Scene}

In order to test the enhanced sensor, orbit, and Earth geometry models a simulated scene was constructed representing a significant fraction of a Landsat image. The scene was designed to simulate the complete Landsat 8 image product (i.e., OLI and TIRS data). Therefore, the spatial fidelity had to span from the OLI $15 \mathrm{~m}$ panchromatic and $30 \mathrm{~m}$ multispectral to the $100 \mathrm{~m}$ TIRS bands (which are resampled and registered to the OLI $30 \mathrm{~m}$ pixels in final products). In addition, the spectral data needed to cover the visible through the long wave infrared range of the two instruments was implemented into the simulated scene. This was accomplished by projecting high resolution $(4 \mathrm{~m})$ satellite multispectral data onto the facetized DEM to generate multispectral texture maps. This was supplemented in the LWIR with ASTER derived emissivity texture maps [8]. These data were also used to generate simple land cover maps (9 classes). Registered AVIRIS data over a large portion of the site were inverted to reflectance and the land cover maps used to generate spectral reflectance/emissivity libraries for each land cover class. DIRSIG uses the combination of land cover type, local texture and a spectral reflectance/emissivity library to assign a spectral reflectance to each sub-pixel sample point [9]. In addition, each land cover class is assigned thermodynamic properties (e.g., specific heat, conductivity, etc.) needed to drive the DIRSIG thermal model. Note that the terms the model is most sensitive to (i.e., solar absortivity, emissivity, slope, and azimuth) are computed on a sub-pixel, per sample basis using spectral library data and the DEM [10]. Figure 4 is an example data set showing a simulated TIRS band of a small subsection of the image where two arrays meet. Thus 
one array is imaging slightly ahead of the satellite ground track and one is imaging slightly behind. To simulate this scene a TLE orbital description for Landsat 7 was used to "fly" the satellite. The nominal clock rates and Earth-centered pointing planned for OLI were used to sample and orient the sensor. Also shown in Figure 4 are an actual Landsat 7 thermal image (top right) and a TIRS band 1 image after alignment (bottom right) illustrating the radiometric similarities. Note that no significant effort was made to match the modeled actual meteorological or phenological conditions to improve the match.

Figure 4. Example data set (left) showing a simulated TIRS band of a small subsection of the Lake Tahoe simulated landscape where the two arrays meet. One array is imaging slightly ahead of the satellite ground track and one is imaging slightly behind. Also, an actual Landsat 7 thermal image (top right) and a simulated TIRS band 1 image after alignment but before geo-rectification (bottom right) are shown to illustrate the radiometric similarities.
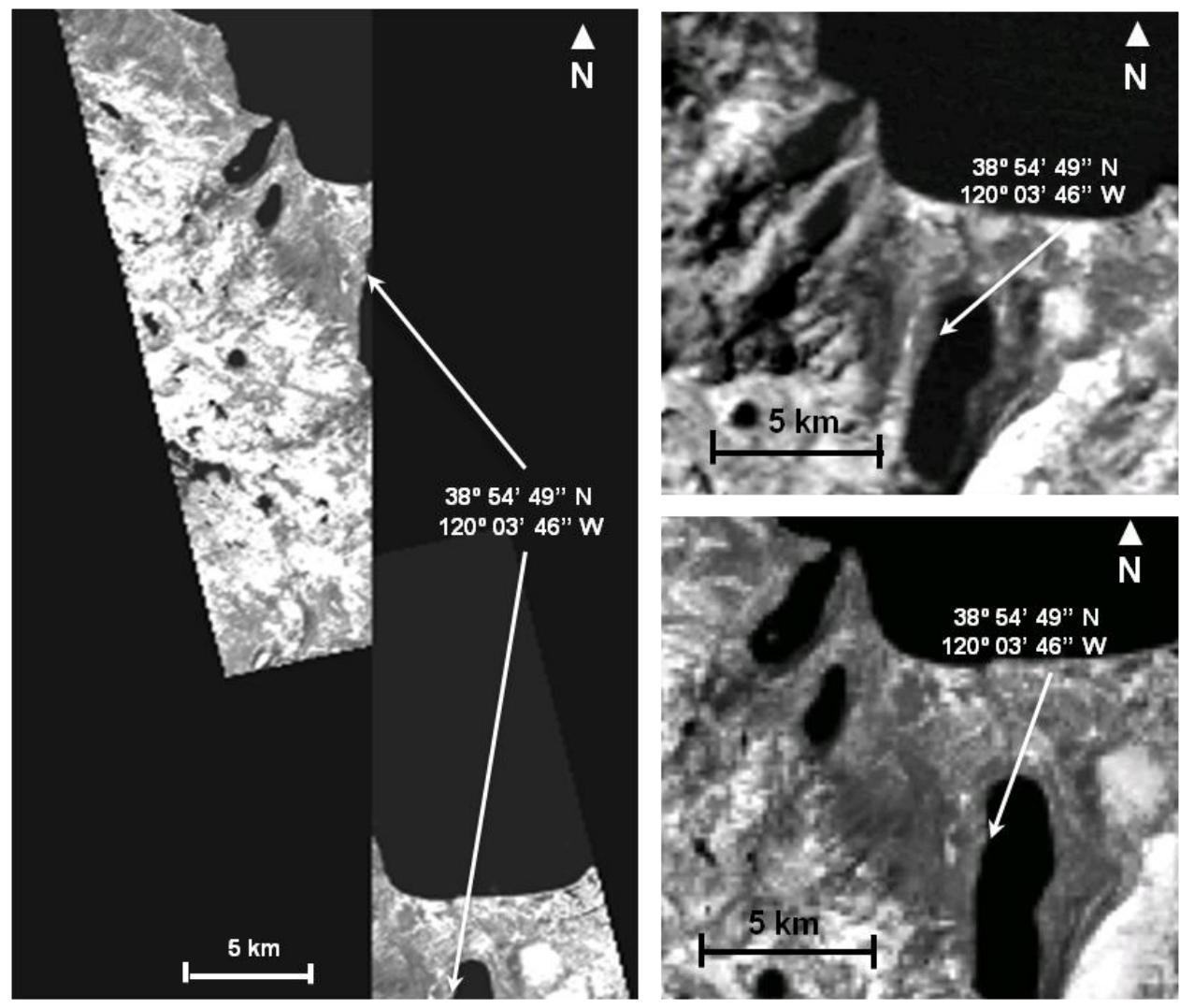

Having upgraded the DIRSIG model, built a Landsat specific sensor model and a scene with Landsat TIRS relevant features, the TIRS instrument team turned to simulation and modeling to help understand and address a number of critical issues that arose during instrument build and test.

\subsection{Simulation of the Potential Impact of TIRS Edge Response Waiver}

The TIRS spatial resolution is largely characterized by the shape of the edge spread function (i.e., how the sensor responds to an edge transition from low radiance to high). During the ground testing of the TIRS instrument an edge is introduced using an edge with a hot background behind it at the focal plane of a collimator. The collimator projects the radiance field from the edge into the TIRS entrance 
aperture to simulate what an edge on the Earth (i.e., very far away) would look like. The TIRS sensor forms an image of the edge from which the edge spread function (ESF) can be calculated (see Figure 5).

Figure 5. Illustration of an edge spread function (ESF) calculated with the TIRS instrument when observing an edge transition between low and high radiance targets. The blue line illustrates the perpendicular to the edge that was used to calculate the edge spread function.

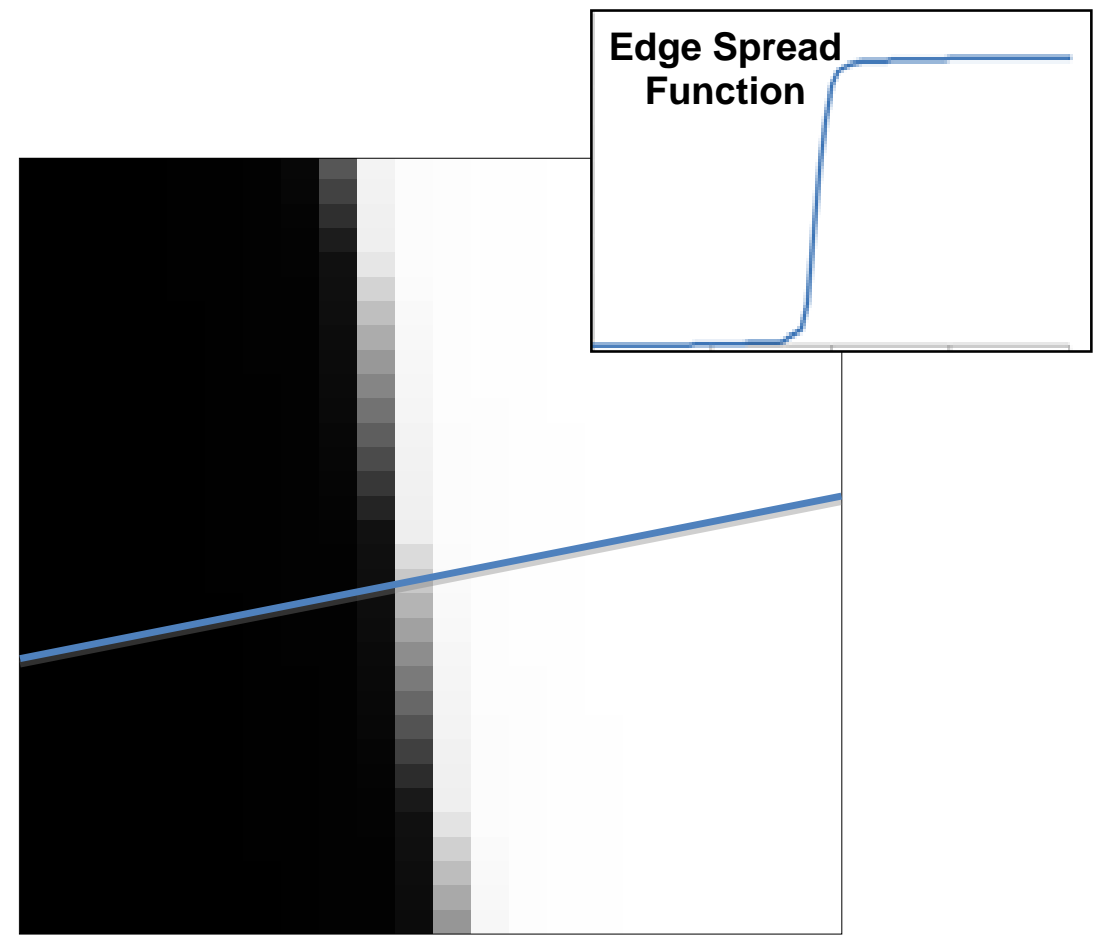

During testing it was observed that the TIRS instrument did not meet the specified edge slope which was designed to control its resolution. To test the impact of this discrepancy, two sets of DIRSIG images were produced. One set of images was produced with the specified edge slope (edge spread function) and the other with the observed edge spread function. These scenes were simulated by generating over-sampled images $(5 \times 5$ samples per pixel $)$ in DIRSIG and then convolving the over-sampled image with the point spread function (PSF) associated with each ESF. Note, the one dimensional PSF is the first derivative of the ESF [4].The convolved image is then down-sampled to the size of the TIRS image. To test the impact of the ESF variation, a scene with large test targets arrayed in Lake Tahoe was produced (see Figure 6). The targets had temperature differences with respect to the lake of $-10 \mathrm{~K}$ to $60 \mathrm{~K}$ in steps of $10 \mathrm{~K}$. Temperature difference image maps produced from images with the specified and observed ESFs are also shown in Figure 6. These data show that significant thermal change induced by the potential blurring associated with the discrepancy from the specified ESF only occurs at extremely high contrast (unrealistically high for most targets). These simulations were generated through four different MODTRAN standard atmospheres. The mid-latitude winter atmosphere produced the greatest apparent temperature difference between the specified and observed ESF images. The minimum, maximum, mean, and standard deviation of the mid-latitude winter case was $0.00 \mathrm{~K}, 1.04 \mathrm{~K}, 0.15 \mathrm{~K}$, and $0.15 \mathrm{~K}$, respectively.

At the time this study was conducted, the discrepancy between the specified and observed ESF was believed to be due to a slight blurring of the input edge introduced by the ground support equipment 
(GSE). The TIRS program granted a waiver on TIRS meeting the edge response based on the assessment that the issue was likely due to GSE issues not instrument issues and due to the very limited impact, if it was an instrument issue, as demonstrated by the simulated data (see Figure 6).

Figure 6. Apparent temperature difference map (in units of Kelvin) between the specified ESF image and the observed ESF image for the $10.8 \mu \mathrm{m}$ band (center) and the $12.0 \mu \mathrm{m}$ band (right) through a MODTRAN mid-latitude summer atmosphere. The test targets located in the lake had the thermal and material properties illustrated (left).
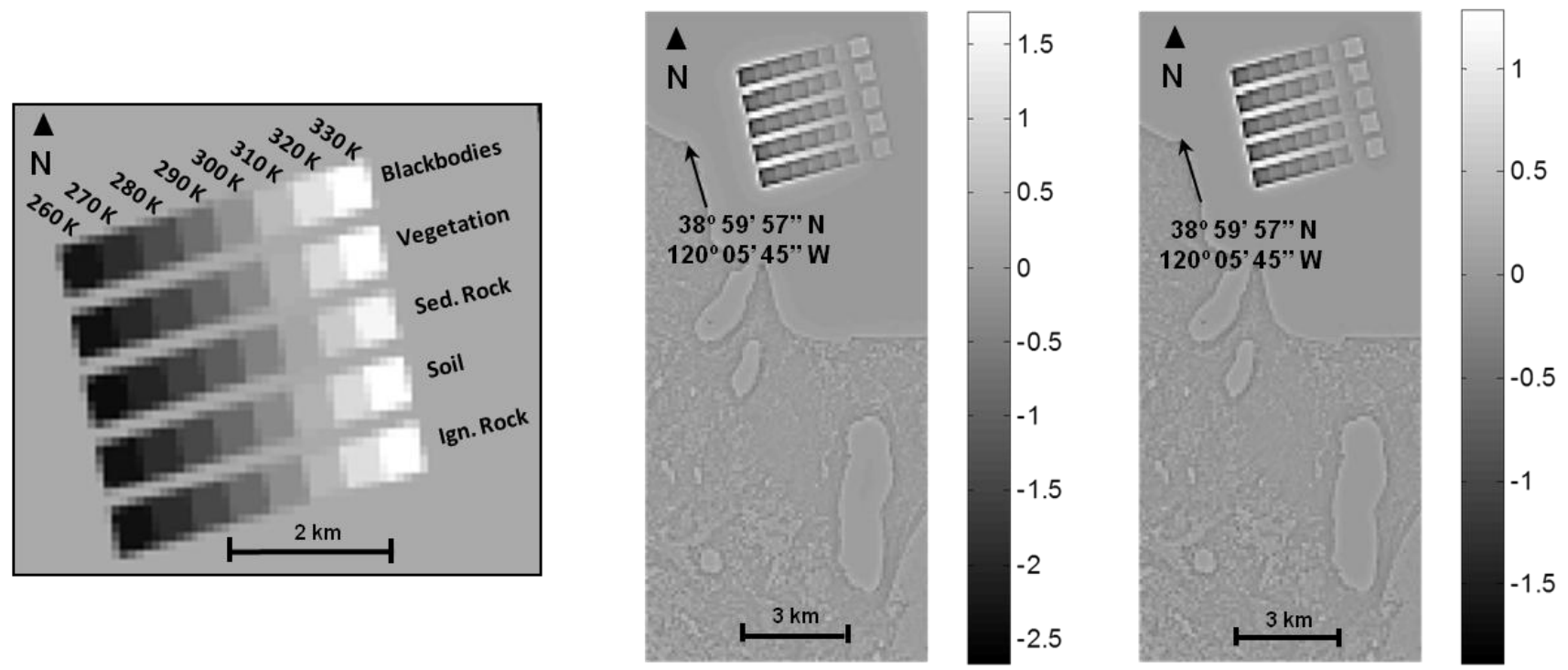

\subsection{Impact of TIRS Cryocooler on Image Quality}

During the design and early testing of the TIRS cryocooler there was concern that the exported vibration from the cryocooler would induce jitter into the TIRS or OLI instrument lines of sight to an extent where unacceptable image blur might result. To assist in evaluating these impacts, DIRSIG was modified to accept both random and correlated jitter spectra as inputs to its pointing model. The jitter spectra are converted in the model to instantaneous samples of the deviation of the optical axis in the in-track and across-track angular directions. The deviations in the optical axis are then used to adjust the optical axis of the simulated sensor each time an image is acquired. However, because the optical axis moves (due to jitter and sensor motion) during the time the photons are being collected during a sample, blurring occurs due to the within-cycle motion as well as geometric distortion due to movement between clock cycles. To account for this, DIRSIG was set to over-sample each pixel spatially and temporally (11 times in $\mathrm{x}$ and y and 11 times temporally). The 11 temporally over-sampled images are then convolved and resampled spatially and temporally to provide the final simulated TIRS image. Figure 7 shows a simulated OLI image produced during algorithm development and testing. The induced jitter for this test had a single low frequency high amplitude mode in the across-track direction resulting in the wavy appearance of the perfectly straight bridge. Note that for these simulations, bridge targets were added to Lake Tahoe in roughly the along and cross-track directions to simulate the targets used operationally to evaluate Landsat spatial resolution [11]. Figure 7 also shows input jitter spectra from a more realistic case (provided by NASA) and spectra computed from the 
DIRSIG generated angular data to verify that pointing data were being correctly simulated. In these simulations two sources of jitter were simulated, the background spacecraft jitter plus the incremental jitter induced by the TIRS cryocooler [12].

Figure 7. Left column shows simulated OLI imagery produced during algorithm development and testing. A high magnitude jitter was applied in the cross-track direction for visual comparison to the "no jitter" case. The right column shows a validation of the DIRSIG jitter model by comparing the input spectra to the spectra derived from the DIRSIG generated angular data (Note the data sets sit on top of each other).
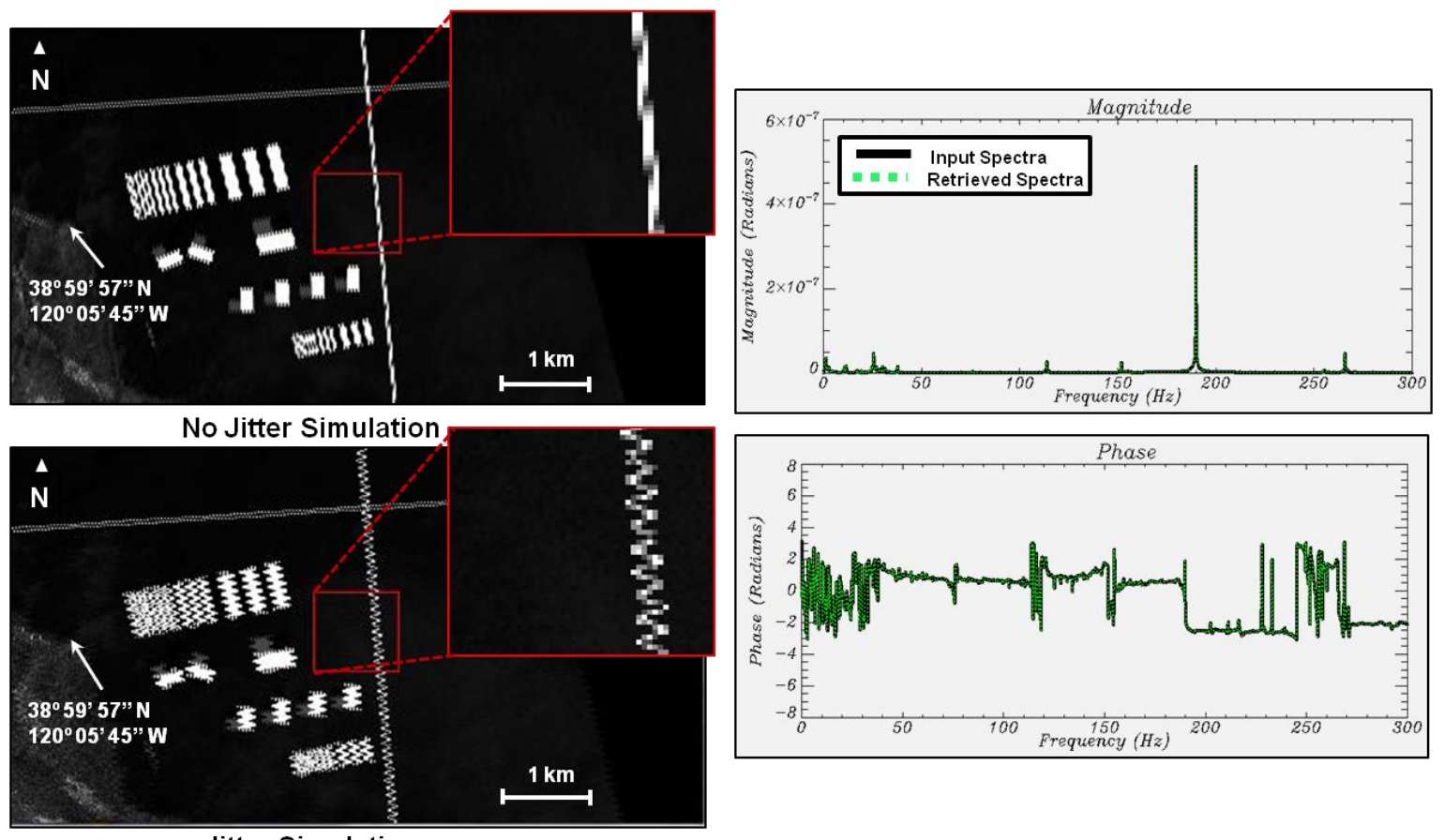

Jitter Simulation

Figure 8 shows some results from the jitter simulations. We utilized a common image based MTF measurement technique [13] to quantitatively assess the impact of the modeled jitter at various levels. The left hand side plot of Figure 8 shows the absolute MTF for the various jitter scenarios modeled while the right hand side plot shows the MTF impact of each jitter scenario relative to the no jitter case. We found the MTF impact, as measured from the simulation image products, to closely follow a Gaussian shape commensurate with the root-mean-square level of jitter [14] for each scenario.

Note the results are shown for the effects of the TIRS cryocooler on OLI since TIRS, with its much larger pixels $(100 \mathrm{~m}$ vs. $30 \mathrm{~m})$, was not significantly impacted by even the high jitter levels. The images with various levels of jitter were provided to the NASA and USGS image assessment teams for evaluation. In the end, to avoid these jitter concerns, further engineering improvements were made to dampen the cryocooler induced vibrations and no significant impacts of jitter on image quality are expected. However, the jitter modeling and all the other DIRSIG improvements are available to support the image assessment teams to help understand and hopefully mitigate any issues that arise in the imagery after launch. 
Figure 8. MTF calculated from "bridge" edges in the OLI simulated scenes for the "no jitter' and the 'TIRS jitter $\times 5$ ' case (left). The jitter impact is more clearly seen in the relative (i.e., ratio to no jitter) plots (right).

Absolute MTF measured along-track

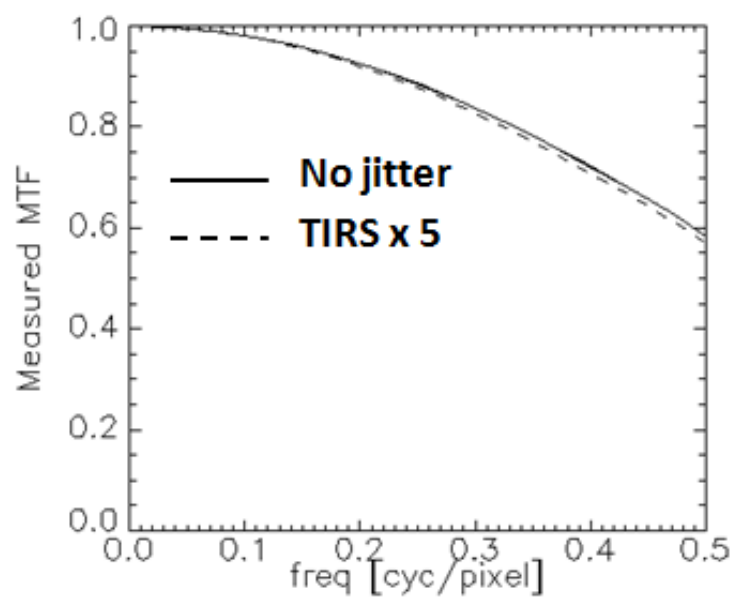

Relative MTF impact of TIRS induced jitter

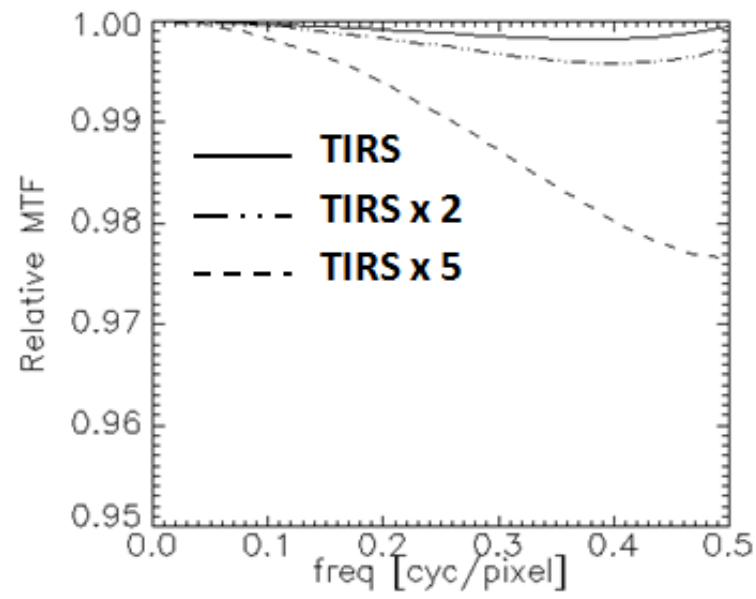

\subsection{Impact of Relative Spectral Response (RSR) Variation on TIRS Streaking and Banding}

To achieve the relatively wide angle $\left(15^{\circ}\right)$ coverage of the TIRS push-broom design, three linear arrays are employed (see Figure 1). Each array has a slightly different relative spectral response (RSR) resulting from slight differences in the manufacturing of the detectors and spectral filters. These differences can result in array to array banding effects. In addition, each detector element within an array can have slight RSR differences resulting in detector-to-detector variations or streaking. Flat-fielding procedures are designed to minimize banding and streaking by forcing the response of all detectors to the same two levels for a hot and cold blackbody target (i.e., detector by detector bias and gain adjustment). This is accomplished on TIRS with a full aperture blackbody that can be set at different levels and by periodic deep space looks. Regrettably, these procedures are only completely effective if the RSRs are truly identical or the targets all have the same spectral shape as the blackbodies. Since neither of these cases is true some banding and streaking will occur. To understand the maximum impact of banding and streaking expected from TIRS, DIRSIG was used to simulate images of a range of targets observed through a variety of atmospheres (note the atmosphere introduces a great deal of spectral shape, often more than material differences, in the LWIR). Recent improvements to DIRSIG allow the user to assign individual RSRs to each detector element as well as detector specific gains and bias values such that the final output can be expressed as:

$$
D C_{i j}=Q\left[g_{i j} G_{j}\left(\frac{\int L_{\lambda i j} R S R_{i j} d \lambda}{\int R S R_{i j} d \lambda}\right)+B_{j}+b_{i j}+n_{i j}\right]
$$

where $D C_{i j}$ is the digital count from the $i^{\text {th }}$ detector in the $j^{\text {th }}$ array [counts];

$Q$ indicates the quantization process (12 bits for TIRS) [counts/volt];

$G_{j}$ is average gain of the $j^{\text {th }}$ array $\left[\operatorname{volts} /\left(\mathrm{w} \cdot \mathrm{cm}^{-2} \cdot s r^{-1} \cdot \mu \mathrm{m}^{-1}\right)\right]$; 
$g_{i j}$ is the relative gain of the $i^{\text {th }}$ detector compared to the array average for array $j$ [unitless];

$L_{\lambda i j}$ is the spectral radiance reaching the $i^{\text {th }}$ detector in the $j^{\text {th }}$ array computed by DIRSIG $\left[\mathrm{w} \cdot \mathrm{cm}^{-2} \cdot s r^{-1} \cdot \mu \mathrm{m}^{-1}\right]$;

$R S R_{i j}$ is the relative spectral response of the $i, j^{\text {th }}$ detector;

$B_{j}$ is the average bias of the $j^{\text {th }}$ array [volts];

$b_{i j}$ is the deviation of the bias of the $i^{\text {th }}$ detector in an array $j$ from the array average [volts];

$\lambda$ is the wavelength $[\mu \mathrm{m}]$ and

$n_{i j}$ is the detector specific noise [volts].

During the development and test of the TIRS instrument, the RSR values for individual detector elements were determined using a spectrometer and a collimator designed to fill the entrance aperture. These RSR values were provided by NASA Goddard based on early testing and used in selecting arrays and evaluating expected image quality relative to banding and streaking effects. The test scene shown in Figure 9 includes a range of targets with varying emissivities set at a wide range of temperatures (see Figure 6).

Figure 9. Percent differences between the two mean class shapes in the $10.8 \mu \mathrm{m}$ channel (top row) and $12.0 \mu \mathrm{m}$ channel (bottom row). From left to right are tropical, mid-latitude summer, mid-latitude winter, and sub-arctic summer atmospheres. Maximum scene differences between the two band shapes are approximately $0.1 \%$ and a $0.2 \%$ for the $10.8 \mu \mathrm{m}$ and $12.0 \mu \mathrm{m}$ channel, respectively.
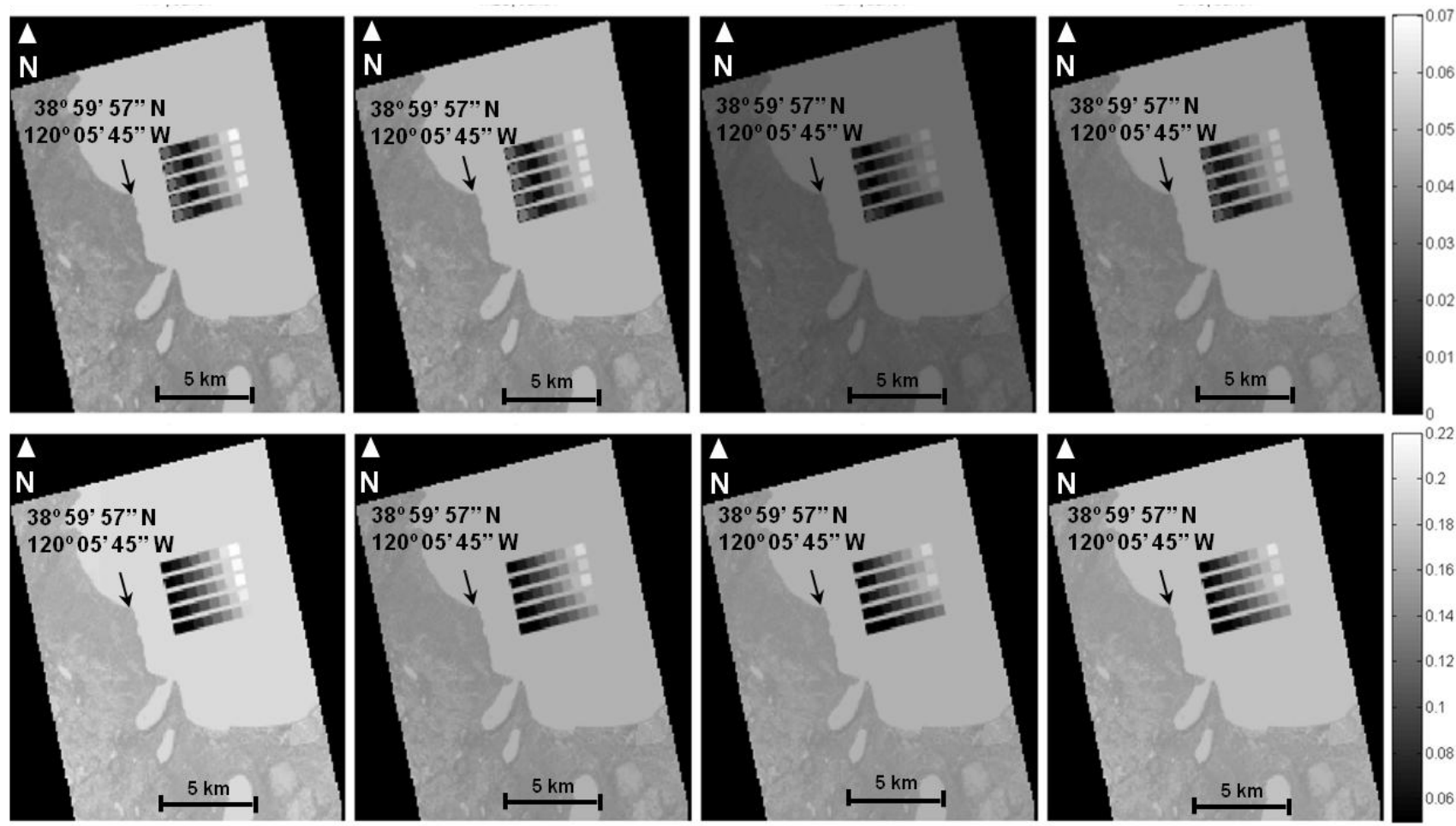

The band shapes of all detectors within a spectral band were grouped into classes. The mean band shape of the two most different classes were provided by NASA Goddard to apply to the synthetic scene. To determine the effect of the two different band shapes within a TIRS channel, each class mean band shape was applied separately to every detector on the DIRSIG scene. In addition, each band 
shape was run with four MODTRAN standard atmospheres assigned to the DIRSIG scene. Every combination of class mean/band shape (two shapes for each TIRS channel) and MODTRAN scene atmosphere (tropical, mid-latitude summer, mid-latitude winter, and sub-arctic summer) yielded 16 DIRSIG scene images. These radiance images were then expressed as apparent temperatures. The percent difference between the two resulting temperature images for a given TIRS channel and for a particular atmosphere were calculated (see Figure 9). These differences represent the difference that would occur at the boundary between two arrays. In general, the differences between the two band shape classes can be expected to produce approximately a $0.1 \%$ and a $0.2 \%$ temperature difference among various materials for a range of atmospheres for the $10.8 \mu \mathrm{m}$ and $12.0 \mu \mathrm{m}$ channels, respectively [15].

\subsection{Potential Use of Side Slither Maneuvers for Flat Field Calibration}

As indicated in Section 2.6, the planned method for flat field calibration (calculation of detector gain and biases to minimize banding and streaking) is to use the on-board full aperture blackbody. As a risk mitigation effort, an initial study was conducted to evaluate the potential to use data collected during a side slither maneuver to supplement, or replace if necessary, the on-board calibrator. In this maneuver, the satellite is yawed $90^{\circ}$ such that to first order all the detectors in an array image over the same line of points on the Earth [16]. Thus, over time each detector sees the same target. In practice this maneuver is done over relatively uniform targets and many samples (e.g., 1,000) in the in-track line are averaged. By averaging the same 1,000 pixel region on the Earth as seen sequentially by each detector, we generate signals (digital counts) that "should" be the same for each detector. First, the dark (low radiance) values are bias adjusted to a common value based on a deep space look. Then the relative gains can be corrected to force the signals to a common value according to

$$
g_{i j}=\frac{\overline{D C}_{j}}{\overline{D C}_{i j}}
$$

where $\overline{D C}_{i j}$ is the average digital count for the side slither samples from the $i^{\text {th }}$ detector in the $j^{\text {th }}$ array and $\overline{D C}_{j}$ is the average of all the $\overline{D C}_{i j}$ values in the $j^{\text {th }}$ array.

Using the relative gains calculated according to Equation (2), streaking effects should be removed. If the arrays look at features on the ground that have the same radiance on average then an array-to-array relative gain correction can also be calculated in a similar fashion. Note, that this is complicated by the fact that in side slither mode the before and aft looking arrays are now looking slightly east or west of the nominal ground track at different lines of targets on the ground (see Figure 10). Thus, this requires imaging of very homogeneous scenes. Another alternative is to perform array-to-array relative gain corrections by taking advantage of the roughly 28 pixel overlap in the ground track between the edges of adjacent arrays in the normal push-broom mode (see Figure 1).

For this study, variation in the individual detector electronic gain values was simulated (deep space is used to get a background measurement to subtract off the instrument self-emitted signal). Side slither data were then simulated using DIRSIG to "fly" the TIRS sensor over a scene with actual Landsat thermal data "glued" to the Earth. This was done to evaluate the spatial uniformity of the radiance from the Earth in terms of its suitability for side slither based flat fielding. 
Figure 10. Illustration of the ground track of two TIRS arrays when imaging in side slither mode. The dark lines represent the ground tracks for a fore and aft looking array superimposed on a Landsat 5 image.

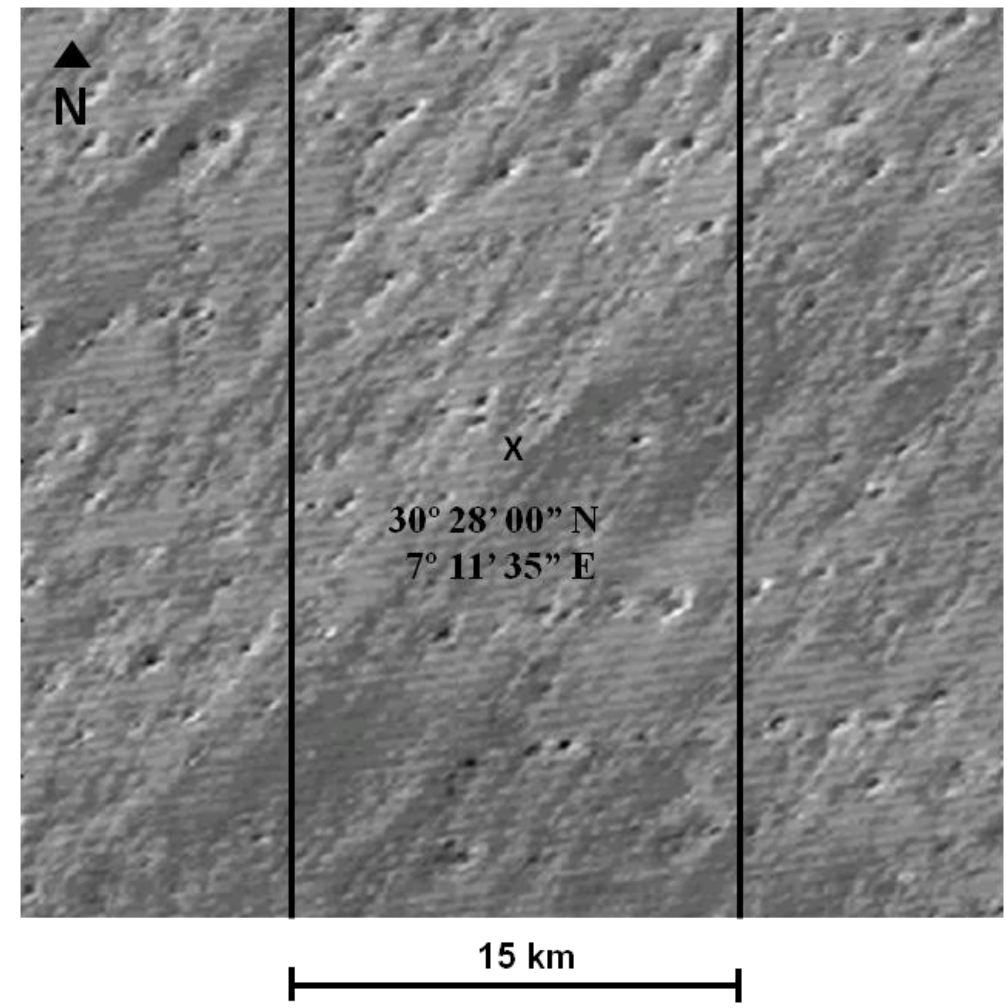

For this simulation, only 300 detectors on one of the TIRS arrays were simulated. Each detector was assigned a unique RSR, gain, and bias derived from actual pre-flight measurements. A simulated radiometric drift was applied by randomly varying the gain and bias of each detector. The side slither data was then utilized to develop a relative correction for the detectors to reduce the striping artifact. Figure 11 demonstrates an original simulated scene without radiometric drift along with striping that occurs due to induced random per-detector drift. The relative correction based on the side-slither data is then applied to significantly reduce the striping [17].

Figure 11. DIRSIG radiance scene of Algeria (left). Radiance image calibrated using preflight radiometric coefficients (center). Radiance image calibrated using pre-flight coefficients with relative correction derived from side-slither data (right).
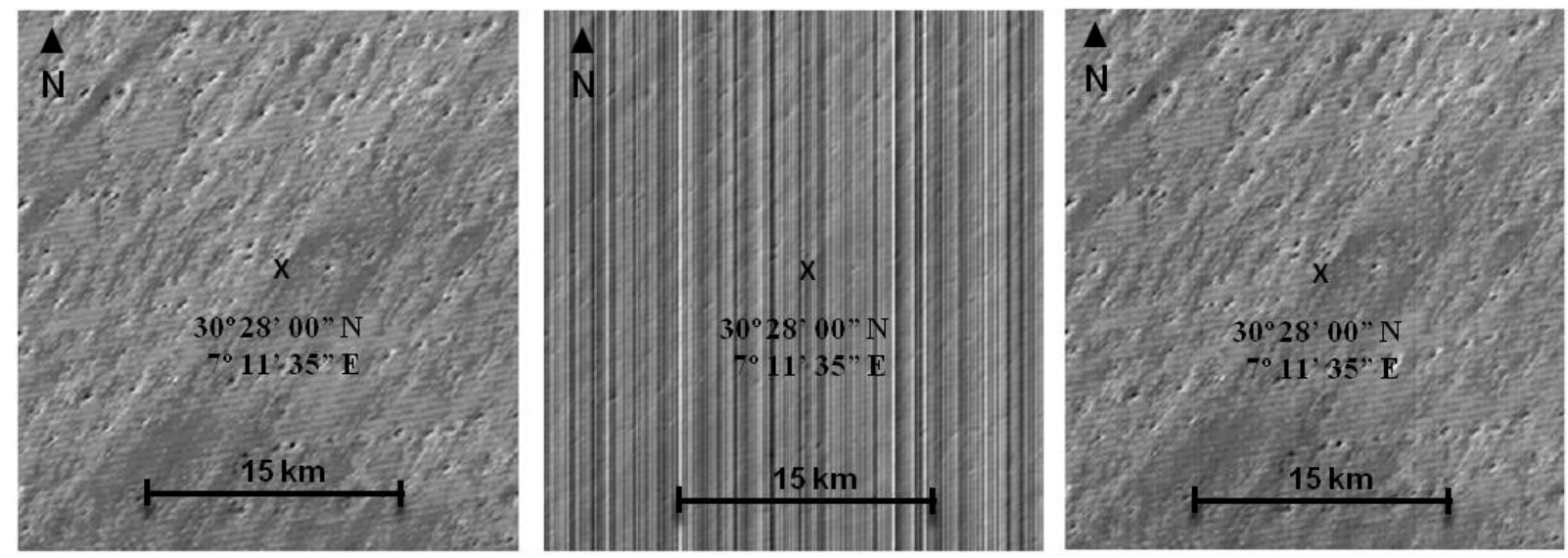
This simulation was performed as a proof of concept for only a subset of the TIRS focal plane. Atmospheric effects were also neglected in this study. Future work will expand this simulation to include the three staggered focal plane arrays and will also include atmospheric and BRDF effects.

\section{Conclusions}

The studies presented here have demonstrated how a synthetic image generation tool has been modified to provide detailed simulation of the performance of the TIRS sensor. The several examples cited show how such a detailed simulation capability can be and was used by an instrument development team to support design trade-off and engineering trade studies. In these studies the simulations showed that the potential degradation in the edge slope from the specified value could induce worst case (for a $60^{\circ} \mathrm{K}$ edge) errors of $1.05 \mathrm{~K}$ (with $0.15 \mathrm{~K}$ the mean expected value). The simulations of TIRS induced jitter on OLI showed that the MTF could be reduced by more than $2 \%$ (worst case) and contributed to the decision to improve the cryocooler dampening. Note, tests showed the DIRSIG jitter model implementation could reproduce the predicted jitter to within numerical accuracy. The TIRS array to array RSR differences were shown to induce acceptable apparent temperature differences at array boundaries of $0.2 \%$ or less for the worst case atmospheric conditions and worst spectral band.

Ongoing work is targeted at using modeling to support the continued development of in-scene calibration techniques. Another problem faced by the Landsat team is the cross-registration of OLI and TIRS datasets. To support this effort, DIRSIG simulations of potential target scenes are being developed and simulated imagery from both sensors will be generated with known pointing and jitter errors to determine what targets have sufficient correlation to enable assessment of the pointing errors. Finally, we expect to use the DIRSIG tools to support the NASA and USGS image assessment teams to simulate and attempt to understand instrument anomalies when the instruments are in orbit.

\section{References}

1. Irons, J.R.; Dwyer, J.L. An overview of the Landsat Data Continuity Mission. Proc. SPIE 2010, doi: 10.1117/12.850416.

2. Reuter, D.; Richardson, C.; Irons, J.; Allen, R.; Anderson, M.; Budinoff, J.; Casto, G.; Coltharp, C.; Finneran, P.; Forsbacka, B.; et al. The Thermal Infrared Sensor on the Landsat Data Continuity Mission. In Proceedings of the 2010 IEEE International Geoscience and Remote Sensing Symposium, Honolulu, HI, USA, 25-30 July 2010; pp. 754-757.

3. Jhabvala, M.; Reuter, D.; Choi, K.; Jhabvala, C.; Sundaram, M. QWIP-based thermal infrared sensor for the Landsat Data Continuity Mission. Infrared Phys. Techn. 2009, 52, 424-429.

4. Schott, J.R. Remote Sensing: The Image Chain Approach, 2nd ed.; Oxford University Press: New York, NY, USA, 2007.

5. Schott, J.R.; Brown, S.D.; Raqueño, R.V.; Gross, H.N.; Robinson, G. An advanced synthetic image generation model and its application to multi/hyperspectral algorithm development. Can. J. Remote Sens. 1999, 25, 99-111. 
6. Vallado, D.A.; Crawford, P. SGP4 Orbit Determination. In Proceedings of AIAA/AAS Astrodynamics Specialist Conference and Exhibit, Honolulu, HI, USA, 18-21 August 2008; AIAA 2008-6770.

7. Schott, J.R.; Raqueño, R.V.; Raqueño, N.G.; Brown, S.D. A Synthetic Sensor/Image Simulation Tool to Support the Landsat Data Continuity Mission (LDCM). In Proceedings of ASPRS 2010 Annual Conference, San Diego, CA, USA, 26-30 April 2010.

8. Hulley, G.C.; Hook, S.J.; Baldridge, A.M. ASTER land surface emissivity database of California and Nevada. Geophys. Res. Lett. 2008, 35, L13401.

9. Scanlan, N.W.; Schott, J.R.; Brown, S.D. Performance analysis of improved methodology for incorporation of spatial/spectral variability in synthetic hyperspectral imagery. Proc. SPIE 2003, 5159, 319-330.

10. Ward, J.T.; Schott, J.R.; Sanders, N.J.; Brown, S.D. Driving Realistic Texture in Simulated Long Wave Infrared Imagery. In Proceedings of 2008 IEEE International Geoscience and Remote Sensing Symposium, Boston, MA, USA, 6-11 July 2008; Volume 3, pp. 728-731.

11. Storey, J. Landsat 7 on-orbit modulation transfer function estimation. Proc. SPIE 2001, 4540, 50-61.

12. Gerace, A.; Gartley, M.; Schott, J.; Raqueño, N.; Raqueño, R. Data-driven simulations of the Landsat Data Continuity Mission (LDCM) platform Proc. SPIE 2011, 8048, 804815.

13. Burns, P. Slanted-Edge MTF for Digital Camera and Scanner Analysis. In Proceedings of IS\&T 2000 PICS Conference, Portland, OR, USA, March 2000; pp. 135-138.

14. Genberg, V.; Michels, G. Integrated modeling of jitter MTF due to random loads. Proc. SPIE 2011, 8127, 81270H-81270H-9.

15. Montanaro, M.; Reuter, D.C.; Markham, B.L.; Thome, K.J.; Lunsford, A.W.; Jhabvala, M.D.; Rohrbach, S.O.; Gerace, A.D. Spectral analysis of the primary flight focal plane arrays for the thermal infrared sensor. Proc. SPIE 2011, 8048, 804816.

16. Henderson, B.G.; Krause, K.S. Relative radiometric correction of Quickbird imagery using the sideslither technique on-orbit. Proc. SPIE 2004, 5542, 426-436.

17. Montanaro, M.; Gerace, A.D. Tracking non-uniformity in the thermal infrared sensor through pre-launch measurements and simulated on-orbit data. Proc. SPIE 2012, 8390, 83902C.

(C) 2012 by the authors; licensee MDPI, Basel, Switzerland. This article is an open access article distributed under the terms and conditions of the Creative Commons Attribution license (http://creativecommons.org/licenses/by/3.0/). 